\title{
ERECTILE FUNCTION OF BPH PATIENTS UNDERGOING A LASER PROCEDURE COMPARED TO A STANDARD TRANSURETHRAL RESECTION OF THE PROSTATE (TURP): A SYSTEMATIC REVIEW AND META-ANALYSIS
}

\author{
Muhammad Fawzi Zulfikar ${ }^{\mathrm{a}}$, Fikri Rizaldi ${ }^{\mathrm{b}}$, Yudhistira Pradnyan Kloping ${ }^{\mathrm{a}}$,Zakaria \\ Aulia Rahman ${ }^{\text {a }}$ Wahjoe Djatisoesanto ${ }^{a}$

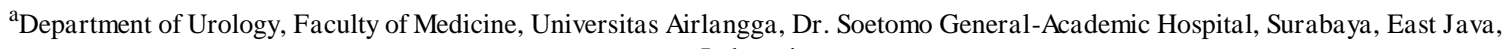 \\ Indonesia \\ ${ }^{\mathrm{b}}$ Department of Urology, Faculty of Medicine, Universitas Airlangga, Universitas Airlangga Teaching Hospital, Surabaya, East Java, \\ Indonesia
}

\begin{abstract}
Transurethral Resection of Prostate (TURP) has been the gold standard of surgical management in Benign Prostatic Hyperplasia (BPH) patients. Various techniques, including laser therapy, have been developed to provide a safe alternative to TURP. The effect of the laser on the patient's erectile function, however, is still unknown. The aim of this study is to evaluate the erectile function of individuals with BPH undergoing surgical intervention with laser procedure compared to the standard TURP procedure. A systematic search was conducted through PubMed and Science-Direct databases up to September 2021. All studies comparing laser therapy to TURP for patients with BPH and reporting the final International Index of Erectile Function (IIEF) and change of IIEF were included in this study. A total of three Randomized Controlled Trials (RCTs) and one retrospective study were included in the final analysis. According to the meta-analysis, laser therapy had a similar final IIEF score during the 12-month follow-up compared to the TURP procedure in the subgroup of IIEF-15, IIEF-5, and the combined analysis ( $p=0.62, p=0.72, p=0.79$, respectively). The change of IIEF from baseline was similar among both modalities $(p=0.8)$. The effect of laser therapy on erection function was similar to the TURP procedure
\end{abstract}

Keywords: Benign prostatic hyperplasia, Transurethral resection of the prostate, Laser therapy, Erectile function 


\section{Introduction}

BPH (benign prostatic hyperplasia) is the most frequent benign tumor in males starting the ages of 40 and beyond [1]. The rate of occurrence rises with age, reaching 90 percent of males in the ninth decade. BPH is described as a change in prostate size that causes Lower Urinary Tract Symptoms (LUTS) in the afflicted population. LUTS can have a significant impact on one's quality of life. A decrease in quality of life and health condition will induce anxiety and desperation, as well as impede everyday activities and movement [2]. LUTS is caused by hyperplasia of glandular and stromal materials in the periurethral and transitional zones, although previous studies have found that there is still controversy about the correlation between the severity of symptoms and prostate size [3]. However, most research concluded that increased prostate size is a positive predictive value of severity of LUTS symptoms. According to one theory, alterations in prostatic receptors such as alpha-adrenergic, muscarinic, and phosphodiesterase-5, as well as anatomical obstruction of the prostate, all contribute to the severity of LUTS symptoms [1].

Therapy of BPH consists of conservative and active management with pharmacological and operative treatment. Patients with no complications and no disruption in quality of life can be offered conservative management. Pharmacological therapy is the first-line therapy in patients with moderate LUTS symptoms and decreasing quality of life. While more invasive therapy is used in patients with more severe LUTS symptoms. Transurethral Resection of Prostate (TURP) has been considered as the gold standard of operative management in $\mathrm{BPH}$ with a prostate volume of $30-80 \mathrm{ml}$ [4]. It is, however, comes with complications such as bleeding, incontinence, fluid body imbalance, and erectile dysfunction. Therefore, more techniques have been developed to provide a safe and effective alternative to TURP, including minimally invasive laser therapy. Various studies reported that laser modality has a similar outcome compared to the routinely performed TURP procedure with the advantage of minimizing the risk of bleeding, reducing the length of hospitalization following the procedure, and shorter catheterization time [5].

Even though the laser modality has some advantages compared to the TURP procedure, the effect of laser on the patient's erectile function is still unknown. The majority of the cohort studies and trials only evaluated the outcome related to LUTS such as IPSS, PVR, and Qmax. Furthermore, trials that evaluated the impact of laser modality on patients' erectile function are still limited, and the combined analysis on the erectile function parameter in both procedures is lacking [6]. Therefore in this article, we aim to perform a systematic review and meta-analysis to evaluate the erectile function of patients with BPH undergoing surgical intervention with laser procedure compared to the standard TURP procedure.

\section{Methods}

This research was carried out in accordance with the recommendations from the Cochrane Handbook for Systematic Review for Interventions and Preferred Reporting Items for Systematic Review and MetaAnalysis (PRISMA) [7,8]. This study protocol was registered in The International Prospective Register of Systematic Review (CRD42021257966) [9].

\section{Systematic search strategy}

A systematic search was conducted through PubMed and Science Direct databases up to September 2021. The keywords used in the systematic search were: "Lasers" OR "Holmium" OR "Thulium" AND "transurethral" AND "resect" AND "prostate" OR "prostatic" OR "prostatitis". Two reviewers conducted the systematic search. Further relevant articles were evaluated by reading the full-text articles using our predefined eligibility criteria 


\section{Eligibility Criteria}

All studies comparing laser therapy to TURP for patients with BPH and reporting the following outcomes: final International Index of Erectile Function (IIEF) score and change of IIEF score. We excluded studies with no English full text, patients with ED before surgery, and inadequate dosing information.

\section{Data Extraction and Quality assessment}

The study selection and data extraction were conducted by two independent reviewers (F.A.W and W.D). Any disputes regarding the conclusion were discussed and settled between the reviewers. Each reviewer assesses the risk of bias using Instrument of Cochrane Risk of Bias Tools 2 for Randomized Trial for Randomized Controlled Trial study. There were 7 domains that were assessed, bias due to confounding, bias due to selection of participants, bias in classification of interventions, bias due to deviations from intended interventions, bias due to missing data, bias in measurement of outcomes, and bias in selection of the reported result. Each of those domains was classified on two judgements, some concerns or low concerns. For observational study, Newcastle Ottawa Scale was used to assess quality. The studies were considered good quality if they met the score equals to or more than 6 in Newcastle Ottawa Scale score

\section{Statistical Analysis}

We assessed heterogeneity between studies using I2 and P-value. The Mantel-Haenszel fixed effects model was selected if there was low heterogeneity (I2<50\%; p-value $>0.05$ ), while the Random-Effects Model was selected if the pooled analysis showed high heterogeneity among the studies (I2 50\%; p-value < $0.05)$ [10]. We presented the pooling analysis of our result in the form of Mean Differences due to our extracted data was in a form of continuous data. We analysed different IIEF instrument using subgroup analysis. All analyses were carried out using statistical software Review Manager 5.4 (Cochrane Collaboration, Oxford, UK)

\section{Results}

\section{Baseline Characteristics}

From the searching through multiple databases as shown in PRISMA flow in figure 1, we obtained a total of 369 records of title and abstract. After compiling the records using Mendeley reference software, we remove 12 duplicates using manual searching and duplication removal tools from Mendeley. After removing the duplicates, we proceeded to screen 357 titles and abstracts and retrieve 113 full-text articles to be assessed. Finally, we found three RCTs and one retrospective study that met our eligibility criteria to be included. A total of 442 patients were included in the final analysis, with an average age of 66.2 years and an average prostate volume of $61.9 \mathrm{ml}$. Two studies compared holmium laser and TURP, while two other studies using Thulium laser modality as the comparison to the TURP. The summary of the baseline characteristics of the included studies is presented in table 1 . 
Table 1. Baseline characteristics of the included studies

\begin{tabular}{|c|c|c|c|c|c|c|c|}
\hline \multirow{2}{*}{ Author } & \multirow{2}{*}{ Design } & \multirow{2}{*}{ Sample } & \multirow{2}{*}{ Intervention } & \multicolumn{2}{|c|}{ Age ( years) } & \multicolumn{2}{|c|}{ Prostate volume (ml) } \\
\hline & & & & Laser & TURP & Laser & TURP \\
\hline Briganti 2006 & $\mathrm{RCT}$ & $60 / 60$ & Holmium vs TURP & $65.25 \pm 6.9$ & $64.18 \pm 7.2$ & $73.3 \pm 31.7$ & $58.2 \pm 21.4$ \\
\hline Montorsi 2004 & RCT & $52 / 48$ & Holmium vs TURP & 65.14 & 64.5 & $70.3 \pm 36.7$ & $56.2 \pm 19.4$ \\
\hline Xia 2008 & RCT & $52 / 48$ & Thulium vs TURP & $68.9 \pm 7.7$ & $69.3 \pm 7.3$ & $59.2 \pm 17.7$ & $55.1 \pm 16.3$ \\
\hline Wang 2014 & Retrospective & $63 / 59$ & Thulium vs TURP & $65.8 \pm 6.3$ & $66.3 \pm 5.8$ & $62.3 \pm 15.8$ & $60.8 \pm 14.3$ \\
\hline
\end{tabular}
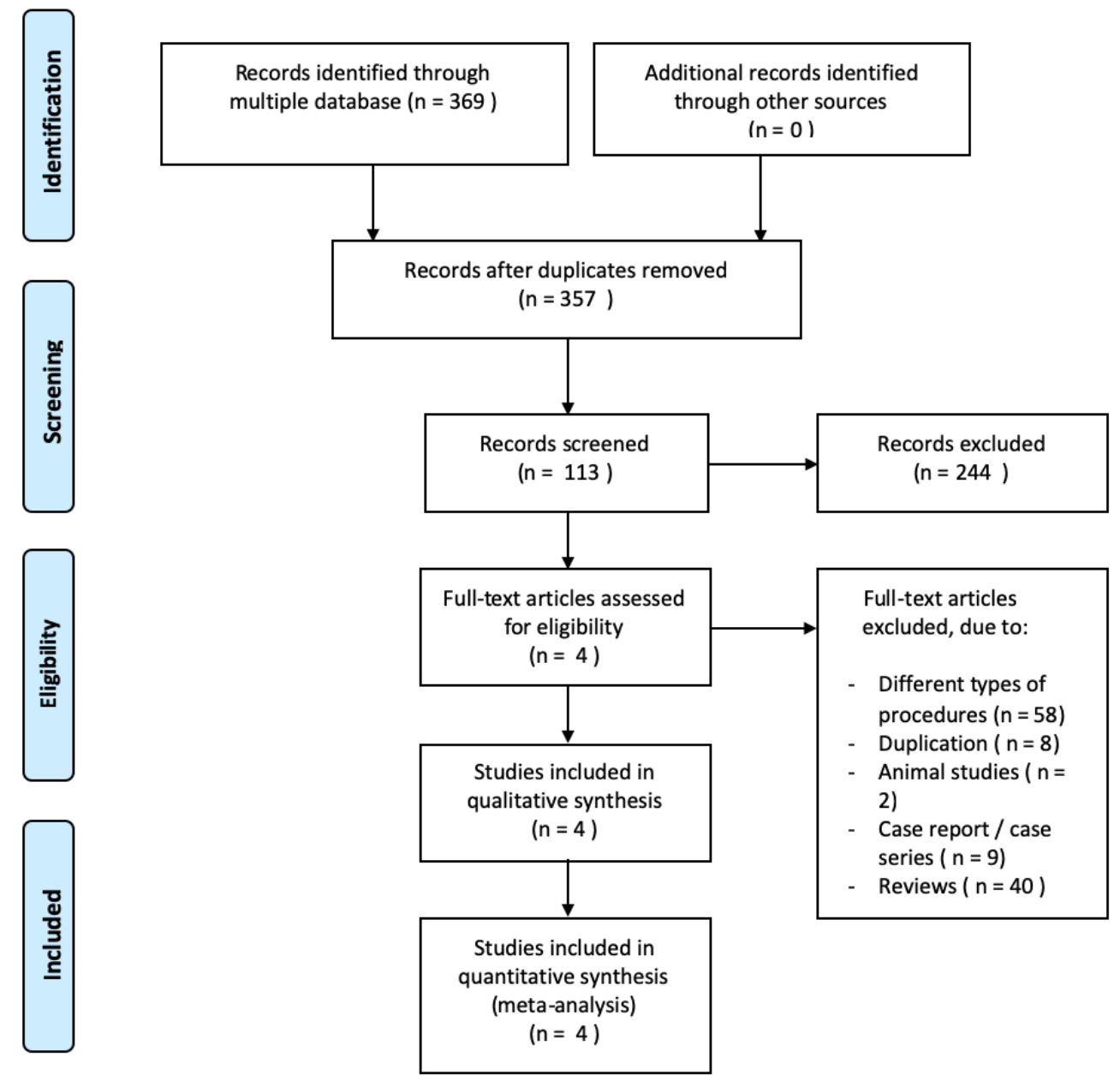

Figure 1. PRISMA Flow diagram 


\section{Quality Assessment}

In this meta-analysis, we included three RCTs and assessed the quality using the Risk of bias tool 2 developed by Cochrane. All of the included trials reported adequate information regarding the randomization process; therefore, risk of bias in the randomization process domain was low. Furthermore, the data regarding missing outcome data, measurement of the outcome, and the selection of the reported result were described in detail. Therefore, the overall Risk of bias among the included trials was low. In this review, we included an observational study that was evaluated using the NOS. From the final assessment, the study by Wang et al.[20] had a score of 7 because the study described the selection, comparison, and outcome with adequate information. The summary of the quality assessment is summarized in Figure 2 for Cochrane Risk of Bias tool 2 and table 2 for Newcastle Ottawa Scale

Table 2. Quality assessment of the included study using Newcastle Ottawa Scale for observational studies

\begin{tabular}{cccccccc}
\hline & & & & & Quality Score \\
\cline { 3 - 7 } No & Author & Year & Design & Selection & Comparability & Exposure & Total \\
\hline 1 & Xia et al. & 2014 & Case control & 3 & 1 & 3 \\
\hline
\end{tabular}

Study ID
Briganti 2006
Montorsi $2004+\infty$

Figure 2. Quality assessment of the included studies using RoB 2 for RCTs study 
The International Index of Erectile Function (IIEF)

In the outcome of erectile function, four studies with 442 participants who underwent surgical modality for BPH were analyzed. Three studies evaluated the erectile function using IIEF-15 score, while one studies using IIEF-5 score. In this erectile function outcome, we conducted the subgroup analysis based on the instrument used in the study. The forest plot on the analysis of the final IIEF score during the 12-month follow-up revealed an insignificant difference between laser modality and TURP using the IIEF-15 ( $\mathrm{p}=$ $0.62)$, IIEF-5 ( $p=0.72)$, and the combined analysis $(p=0.79)$ (Figure 3$)$. The fixed-model effects were used to analyze the study because heterogeneity was observed to be low (I2 $=0 \%$, heterogeneity $\mathrm{p}=0.73$ ). Furthermore, the forest plot analysis on the change of IIEF score from baseline revealed no significant difference in the group treated with laser modality compared to TURP (MD - $0.14 ; 95 \% \mathrm{CI}-1.21,0.93 ; \mathrm{I} 2=$ $0 \% ; \mathrm{p}=0.8$ ) (Figure 4).

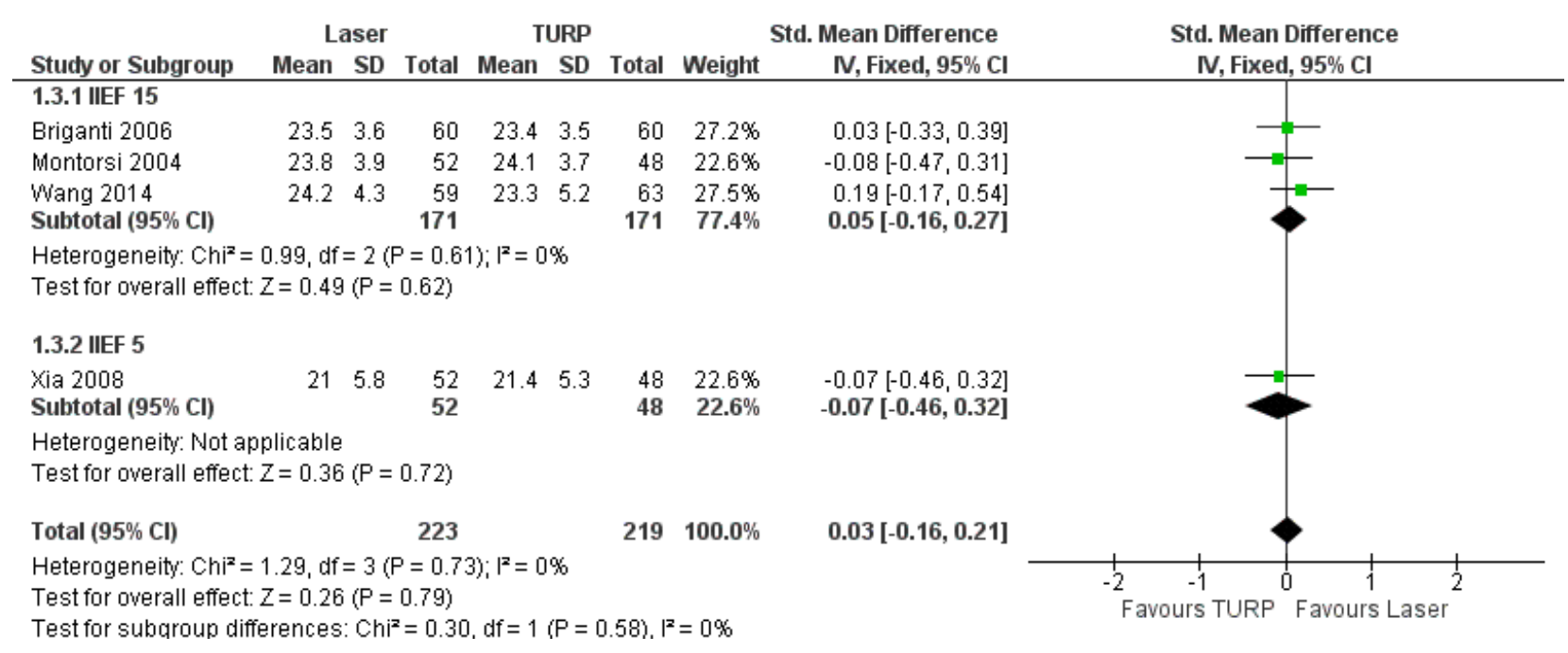

Figure 3. Forest plot on the assessment of final IIEF between laser modality and TURP during 12 month follow-up

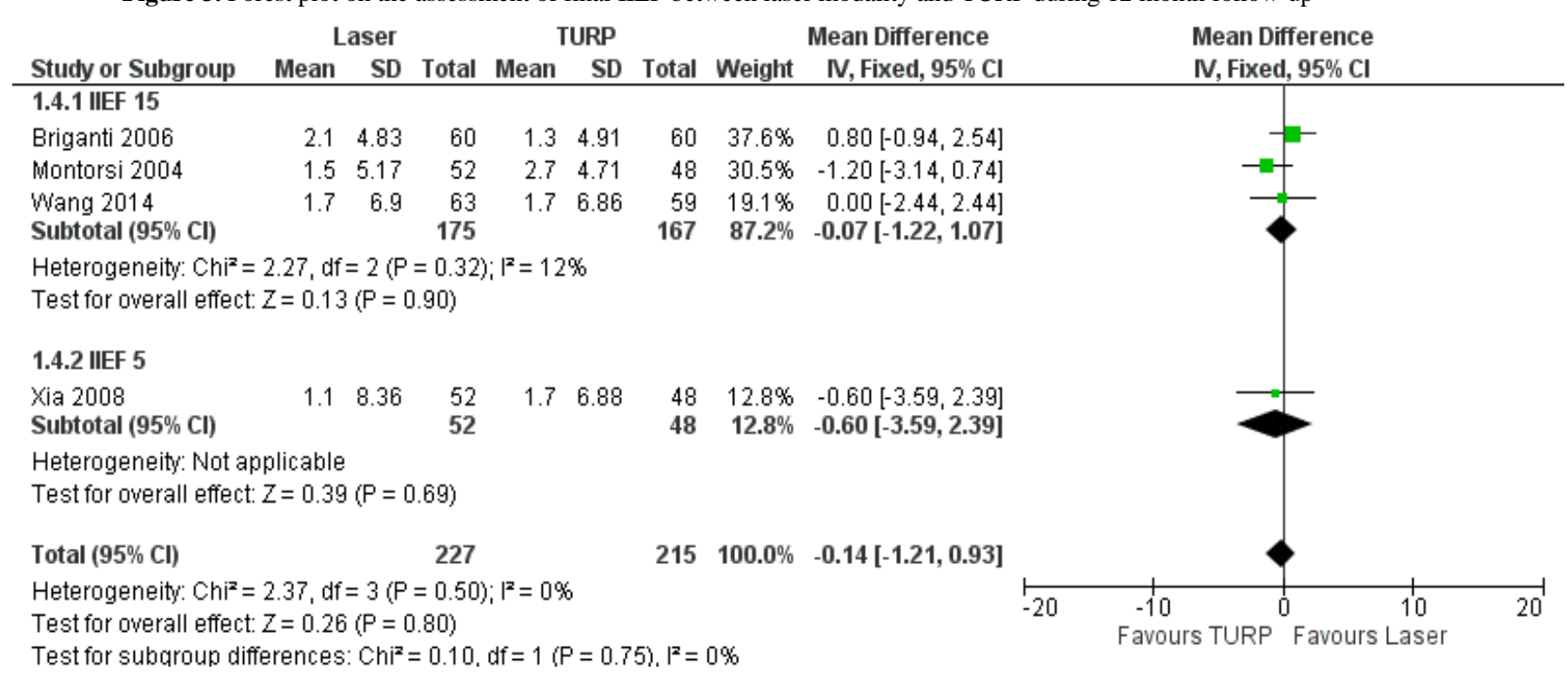

Figure 4. Forest plot on the change of IIEF from baseline between laser modality and TURP during 12-month follow-up 


\section{Discussion}

Benign Prostatic Hyperplasia (BPH) is a benign tumor that most typically affects males in their early forties. As people get older, the prevalence of the disease rises [1]. Lower Urinary Tract Symptoms were more commonly caused by this ailment in men over the age of 50. Although not all men with BPH and LUTS require treatment, this disease can have a major impact on the quality of life of the patient. The problem of severe and bothersome LUTS necessitated medical attention. The decrease on quality of life and health status will impair mobility and daily activity, resulting in discomfort, anxiety, and depression [2].

The benign enlargement of the prostate occurred due to the increase in the number of benign cells in the prostate. BPE is a disease that affects mostly older men, with $50 \%$ of males developing it by the age of 65 and $90 \%$ by the age of 90 . In the fifth decade, the prevalence of moderate/severe LUTS (IPSS > 7) is around $20 \%$. This condition caused the decrease in patient's quality of life [11]. BPH and sexual function have previously been studied to see if there was a cause-and-effect relationship. Several studies have supported up the hypothesis that BPH and erectile dysfunction may coexist [12].

Older age, metabolic, and hormonal variables were all found to be associated to BPE and erectile dysfunction. In a previous study, researchers have found no correlation between prostate size, uroflowmetry, and erectile dysfunction in BPH patients [13]. On the other side, there was previous study which proved the direct correlation between BPE and erectile dysfunction even after adjusting age, medications and comorbidities [14].

In BPH condition, gland and stromal tissue underwent hyperplasia in transitional zone causing LUTS. Although there was no clear relationship between prostate volume and the severity of symptoms, LUTS in $\mathrm{BPH}$ patient was due to static component of prostatic anatomy obstruction and dynamic component which was through receptors in prostate and surrounding tissues including alpha-adrenergic, muscarinic, and phosphodiesterase-5 [1].

LUTS and erectile dysfunction possessed independent association. Degenerative condition could become a predictor of LUTS incidence and erectile dysfunction. Severe LUTS condition could serve as a predictor in determining severity of erectile dysfunction. The more severe LUTS then the higher the probability of more severe erectile dysfunction. Most of BPH and LUTS patient which underwent operative management possessed erectile dysfunction problem [15].

In BPH patients with a prostate volume of $30-80 \mathrm{~mL}$ and daily activity impairment due to LUTS symptoms, transurethral resection of the prostate is still the preferred procedure [4]. Bleeding, electrolyte imbalance, excess fluid absorption, incontinence, and erectile dysfunction were some of the complications that might occur as a result of the TURP operation, which is why a growing range of less invasive techniques for BPH are being used to reduce patient morbidity In this case, laser technology might be a viable alternative to avoid complications [16]. The effect of TURP procedure in erectile dysfunction incidence was still controversial. Previous studies found that the TURP operation had a detrimental effect on BPH patients, causing erectile dysfunction; however, other studies found that the erectile dysfunction condition improved following the procedure. International Index of Erectile Function (IIEF) was an assisting tool which is commonly used to help in the diagnosis of this disease [17].

In this meta-analysis study, laser technology was compared with TURP procedure in patient with BPE. The erectile function was assessed as the outcomes of the comparison. The laser chosen in this study was holmium and thulium laser This decision was made based on the capacity to penetrate superficial prostate tissue, perform enucleation, and extract tissue for histological evaluation. Therefore, in the end, all tissue samples could be confirmed to not possess any cancer cells [16,18-20].

The surgical technique which was used in HoLEP procedure was the morcellation of prostatic tissue lobe into the small fragment that can be extracted from bladder cavity. Both of these studies classified the procedures performed by urologists in two hospital teaching centers into two groups using the HoLEP approach. The Holmium laser radiation was transmitted through a $24 \mathrm{Fr}$ fiber implanted inside the resectoscope. Enucleation was performed using a frequency of $35 \mathrm{~Hz}$ and a power of $2.0 \mathrm{~J}$. Both studies utilized the prostate vapoenucleation procedure $[18,19]$.

There were 2 studies that used Thulium laser included in this study. From the study which was done by 
Xia et al. [16] in 2008, the average power of thulium laser was 50-W which was operated in continuous wave mode. This energy was transferred through the $550 \mathrm{~mm}$ PercuFib endfiring fiber. Laser fiber was inserted through Continous type of Karl Storz Resectoscope with the size of 26 Fr. This procedure was named tangerine technique where the prostatic tissue was resected like peeling the orange [16]. This was different from the study by Wang et al. [20] in 2014 where the technique used was vapoenucleation with thulium laser energy of 120-W and the wave length of 2- $\mu \mathrm{m}$ [20].

For now, there has been debate about whether the operational procedure in patients with BPH-LUTS can improve or worsen erectile function. A recent study found that TURP or laser treatment might impair erectile function. This finding was backed up by two theories. The presence of heat was first transmitted into the posterolateral prostate's neurovascular bundle. The presence of perforation in the prostatic capsule was the second reason. This conclusion contradicted previous research, which claimed that treating BPHLUTS would improve erectile function by reducing LUTS symptoms, allowing patients to regain psychological function and reduce changes in cavernosal body structure caused by persistent BPH-LUTS [21].

The difference of the mean IIEF score domain did not possess statistically significant value $(\mathrm{p}=0.081)$ which the accumulation of mean difference of $0.10(95 \% \mathrm{CI}=0.69-0.88)$. From four studies which was included based on the inclusion criteria, there was no study that showed significant difference between the mean IIEF score erection domain. This is in accordance with the study from Brookes et al.[22] in which there was no difference found in erection function in patient undergoing TURP, laser or watchful waiting [22]. Another study reported that TURP could reduce erection function in around $40 \% \mathrm{BPH}$ patient which underwent TURP, however several patients who experienced the reduction in erection function in the study were the patient that was previously diagnosed with erectile dysfunction and diabetes mellitus [23].

Holmium laser was not proven to reduce erection function or ejaculation. A study done by Briganti et al.[19] reported that BPH treatment using laser therapy could increase erection function but the result was not significant [19]. This finding was in accordance with the study that used Thulium laser in which there was in-significant difference in erection function before and after BPH therapy using Thulium laser [18] In contrast with greenlight type laser, BPH treatment using laser therapy could reduce erection function in several studies. This result was probably due to the penetration ability from greenlight laser which is theoritically deeper compared to holmium or thulium laser [24].

The reduction in erectile function was not only in BPH patient, but also in patient undergoing surgery in this condition. TURP was associated with the risk of post-operative erectile dysfunction. Leliefeld et al. [12] conducted a cohort study from 679 patients with the follow up in early and 9 months after several varieties of treatment for BPH including TURP, medicamentous therapy with finasteride and watchful waiting [12]. Around $84 \%$ patient did not report any changes in sexual function and there was no significant difference found between each intervention group. On the other side, in prospective cohort study, more than $30 \%$ from 1339 male which did not report any difficulty in erection before operation experienced the reduction in erection function 3 months after TURP, open prostatectomy and TUIP [13].

The size of the prostate and operative time between the laser therapy and TURP in this study was not equal. All individual studies showed that prostate size given with laser therapy possessed larger size compared to TURP procedure. Operative time was also longer in laser therapy compared to TURP procedure. The difference of prostate size and operative time could result to a bias and eventually become limitation in this study.

This meta-analysis study reported a non-significant increase in IIEF score in patient with TURP procedure and laser. This result was in accordance with previous RCT which compared sexual function in patient with BPH undergoing TURP, laser therapy and conservative treatment. In those studies, Erection Function (EF) increased significantly after operation in TURP group. Only 5\% patient reported impotence after operative procedure [25]. Moreover, positive correlation between the increase of erection and urination function after operation which was found in several other studies confirmed the hypothesis that the increase in urinary tract symptoms may indirectly associated with the increase of erection function. The reduction in erection function after TURP procedure may be temporary and was caused by thermal injury in nerve or emotional stress [26]. 
This study comprised several limitations. One study which was included in the inclusion criteria was in retrospective design therefore the interpretation of the result should be made carefully. Moreover, not all studies assessed the erection function using IIEF-15 score in which there was 1 study that used IIEF-5 score. To prevent any potential bias from these two different instruments, we performed subgroup analysis to distinguish study that used IIEF-15 and IIEF-5 score. Also, there were several studies that used thulium laser with different techniques which potentially could produce a bias in this study.

\section{Conclusion}

The impact of laser treatment on erection function was comparable to that of the TURP procedure. Therefore, both of these treatments could become the treatment of choice for erectile dysfunction. In patients with BPH, laser treatment and TURP have not been shown to improve erectile function. 


\section{REFERENCES}

1. Langan RC. Benign Prostatic Hyperplasia. Prim Care - Clin Off Pract [Internet]. 2019;46(2):223-32. Available from: https://doi.org/10.1016/j.pop.2019.02.003

2. McVary KT. BPH: Epidemiology and comorbidities. Am J Manag Care. 2006;12(SUPPL. 5):122-8.

3. Simon RM, Howard LE, Moreira DM, Roehrborn C, Vidal AC, Castro-Santamaria R, et al. Does Prostate Size Predict the Development of Incident Lower Urinary Tract Symptoms in Men with Mild to No Current Symptoms? Results from the REDUCE Trial. Eur Urol. 2016 May;69(5):885-91.

4. Gravas S, Cornu JN, Gacci M, Gratzke C, Herrmann TRW, Mamoulakis C, et al. EAU Guideline on Management of Non-Neurogenic Male Lower Urinary Tract Symptoms (LUTS), incl. Benign Prostatic Obstruction (BPO). In: European Association of Urology. 2021. p. 5-52.

5. Hueber PA, Zorn KC. Canadian trend in surgical management of benign prostatic hyperplasia and laser therapy from 2007-2008 to 2011-2012. J Can Urol Assoc. 2013;7(9-10):5-9.

6. Li Z, Chen P, Wang J, Mao Q, Xiang H, Wang X, et al. The impact of surgical treatments for lower urinary tract symptoms/benign prostatic hyperplasia on male erectile function A systematic review and network meta-analysis. Med (United States). 2016;95(24).

7. Moher D, Liberati A, Tetzlaff J, Altman DG TPG. Preferred Reporting Items for Systematic Reviews and Meta-Analyses: The PRISMA Statement. PLoS Med. 2009;6:7.

8. Book Series C, Higgins J, Green S. Cochrane Handbook for Systematic Reviews of Interventions. THE COCHRANE COLLABORATION ®; 2008.

9. Zulfikar MF, Djatisoesanto W. International prospective register of systematic reviews Citation Review question Participants / population Intervention ( $\mathrm{s}$ ), exposure ( $\mathrm{s}$ ) Main outcome ( $\mathrm{s}$ ) Additional outcome ( s ) Risk of bias ( quality ) assessment Anticipated or actual start date. 2021;(1):1-3.

10. Higgins JPT, Thompson SG. Quantifying heterogeneity in a meta-analysis. Stat Med. 2002;21(11):1539_ 58.

11. Madersbacher S, Sampson N, Culig Z. Pathophysiology of Benign Prostatic Hyperplasia and Benign Prostatic Enlargement: A Mini-Review. Gerontology. 2019;65(5):458-64.

12. Leliefeld HHJ, Stoevelaar HJ, McDonnell J. Sexual function before and after various treatments for symptomatic benign prostatic hyperplasia. BJU Int. 2002 Feb;89(3):208-13.

13. Green JS, Holden ST, Bose P, George DP, Bowsher WG. An investigation into the relationship between prostate size, peak urinary flow rate and male erectile dysfunction. Int J Impot Res. 2001 Dec;13(6):3225.

14. Hoesl CE, Woll EM, Burkart M, Altwein JE. Erectile dysfunction (ED) is prevalent, bothersome and underdiagnosed in patients consulting urologists for benign prostatic syndrome (BPS). Eur Urol. 2005 Apr;47(4):511-7.

15. Park HJ, Won JEJ, Sorsaburu S, Rivera PD, Lee SW. Urinary Tract Symptoms (LUTS) Secondary to Benign Prostatic Hyperplasia (BPH) and LUTS/BPH with Erectile Dysfunction in Asian Men: A Systematic Review Focusing on Tadalafil. World J Mens Health. 2013;31(3):193.

16. Xia S-J, Zhuo J, Sun X-W, Han B-M, Shao Y, Zhang Y-N. Thulium laser versus standard transurethral resection of the prostate: a randomized prospective trial. Eur Urol. 2008 Feb;53(2):382-9. 
17. Oka AAG, Duarsa GWK, Novianti PA, Mahadewa TGB, Ryalino C. The impact of prostate-transurethral resection on erectile dysfunction in benign prostatic hyperplasia. Res Reports Urol. 2019;11:91-6.

18. Montorsi F, Naspro R, Salonia A, Suardi N, Briganti A, Zanoni M, et al. Holmium laser enucleation versus transurethral resection of the prostate: results from a 2-center, prospective, randomized trial in patients with obstructive benign prostatic hyperplasia. J Urol. 2004 Nov;172(5 Pt 1):1926-9.

19. Briganti A, Naspro R, Gallina A, Salonia A, Vavassori I, Hurle R, et al. Impact on sexual function of holmium laser enucleation versus transurethral resection of the prostate: results of a prospective, 2-center, randomized trial. J Urol. 2006;175(5):1817-21.

20. Wang Y, Shao J, Lu Y, Lü Y, Li X. Impact of 120-W 2- $\mu$ m continuous wave laser vapoenucleation of the prostate on sexual function. Lasers Med Sci. 2014;29(2):689-93.

21. Oudot A, Oger S, Behr-Roussel D, Caisey S, Bernabé J, Alexandre L, et al. A new experimental rat model of erectile dysfunction and lower urinary tract symptoms associated with benign prostatic hyperplasia: the testosterone-supplemented spontaneously hypertensive rat. BJU Int. 2012 Nov;110(9):1352-8.

22. Brookes ST, Donovan JL, Peters TJ, Abrams P, Neal DE. Sexual dysfunction in men after treatment for lower urinary tract symptoms: evidence from randomised controlled trial. BMJ. 2002 May;324(7345):1059-61.

23. Taher A. Erectile dysfunction after transurethral resection of the prostate: Incidence and risk factors. World J Urol. 2004;22(6):457-60.

24. Park J, Cho SY, Cho MC, Jeong H, Son H. Changes in Erectile Function after Photoselective Vaporization of the Prostate with a 120-W GreenLight High-Performance System Laser: 2-Year Follow-Up. World J Mens Health. 2017;35(3):156.

25. Soderdahl DW, Knight RW, Hansberry KL. Erectile dysfunction following transurethral resection of the prostate. J Urol. 1996 Oct;156(4):1354-6.

26. Tscholl R, Largo M, Poppinghaus E, Recker F, Subotic B. Incidence of erectile impotence secondary to transurethral resection of benign prostatic hyperplasia, assessed by preoperative and postoperative Snap Gauge tests. J Urol. 1995 May;153(5):1491-3. 